\title{
Gene replacement therapy after neuropathy onset provides therapeutic benefit in a model of CMT1X
}

\author{
Kagiava A., ${ }^{1}$ Karaiskos C., ${ }^{1}$ Richter J., ${ }^{3}$ Tryfonos C., ${ }^{3}$ Heslegrave AJ., ${ }^{4}$ Sargiannidou I., ${ }^{1}$ \\ Rossor A., ${ }^{4}$ Zetterberg H., ${ }^{5,6,7,8}$ Reilly M.M., ${ }^{4}$ Christodoulou C., ${ }^{3}$ Kleopa K.A. ${ }^{1,2^{*}}$ \\ ${ }^{1}$ Neuroscience Laboratory and ${ }^{2}$ Neurology Clinics, The Cyprus Institute of Neurology and \\ Genetics and Cyprus School of Molecular Medicine, Nicosia, Cyprus \\ ${ }^{3}$ Department of Molecular Virology, The Cyprus Institute of Neurology and Genetics and Cyprus \\ School of Molecular Medicine, Nicosia, Cyprus \\ ${ }^{4}$ Department of Neuromuscular Diseases, UCL Institute of Neurology and National Hospital for \\ Neurology and Neurosurgery, London, United Kingdom \\ ${ }^{5}$ Department of Neurodegenerative Disease, UCL Institute of Neurology, London, United \\ Kingdom \\ ${ }^{6}$ UK Dementia Research Institute at UCL, London, United Kingdom \\ ${ }^{7}$ Department of Psychiatry and Neurochemistry, Institute of Neuroscience and Physiology, the \\ Sahlgrenska Academy at the University of Gothenburg, Mölndal, Sweden \\ ${ }^{8}$ Clinical Neurochemistry Laboratory, Sahlgrenska University Hospital, Mölndal, Sweden
}

Words in Abstract: 285

Total words (excluding methods and figure legends): 6398

Words in introduction: 522

Words in discussion: 1660

Figures: 8

Supplementary Tables: 4

Number of References: 48

"Correspondence: Prof. Kleopas A. Kleopa, MD

The Cyprus Institute of Neurology and Genetics

Cyprus School of Molecular Medicine

6 International Airport Avenue, P.O. Box 23462,

1683, Nicosia, CYPRUS

Tel: +35722358600

Fax: +35722392786

kleopa@cing.ac.cy 


\begin{abstract}
X-linked Charcot-Marie-Tooth disease (CMT1X), one of the commonest forms of inherited demyelinating neuropathy, results from GJB1 gene mutations affecting the gap junction protein connexin32 (Cx32). We previously showed that targeted expression of virally delivered Cx32 in Schwann cells following a single lumbar intrathecal injection of lentiviral vectors in 2-month old Cx32 knockout $(\mathrm{KO})$ mice can improve the outcome of the progressive neuropathy that develops after 3 months of age, indicating that gene replacement therapy could offer a therapeutic benefit for this disorder. The aim of this study was to examine whether delayed gene replacement therapy after the onset of peripheral neuropathy can provide a therapeutic benefit also after the onset of the disease. We injected the lentiviral vector LV-Mpz.GJB1 into 6-month old Cx32 KO mice in order to assess the expression of $\mathrm{Cx} 32$ in peripheral tissues and confirmed expression of $\mathrm{Cx} 32$ in lumbar roots and sciatic nerves correctly localized at the paranodal myelin areas. A treatment trial where groups of mice received either the LV-Mpz.GJB1 or the LV-Mpz.Egfp (mock) vector showed improved motor performance at 8 and 10 months of age. Furthermore, treated mice showed increased sciatic nerve conduction velocities compared to mock-treated mice, as well as improvement of myelination and reduced inflammation in lumbar roots and peripheral nerves at 10 months of age, along with enhanced quadriceps muscle innervation. We further measured the emerging clinically relevant biomarker neurofilament light (Nfl) levels in blood as an additional outcome measure in treatment groups and found that blood Nfl elevation was also ameliorated in fully treated mice. Thus, intrathecal gene delivery after the onset of peripheral neuropathy still offers a significant therapeutic benefit in this disease model, providing a proof of principle for treating patients with CMT1X.
\end{abstract}

Keywords: inherited neuropathy, gene therapy, Schwann cells, connexin32, CMT1X 


\section{Introduction}

X-linked Charcot-Marie Tooth (CMT1X) disease is one of the commonest inherited neuropathies, characterized by progressive weakness and atrophy of distal limb muscles, loss of reflexes, sensory loss and reduced nerve conduction velocities. Disability increases with age mainly resulting from motor unit loss [1]. CMT1X results from mutations in GJB1, the gene encoding the gap junction (GJ) protein connexin32 (Cx32). In addition to peripheral neuropathy, a number of CMT1X mutations also result in central nervous system (CNS) phenotypes characterized by spasticity, hyperactive reflexes, extensor plantar responses, ataxia, or acute reversible encephalopathy [2-6]. CMT1X affects mostly men with onset at the age of 5 to 20 years $[3,7,8]$, while affected women may present at a later stage $[9,10]$ with milder symptoms [11].

Cx32 forms intracellular GJ channels through the non-compact myelin layers at paranodal loops and Schmidt-Lanterman incisures of Schwann cells $[12,13]$. These channels serve important homeostatic and axon-glial signaling functions in peripheral myelinated fibers. There are over 400

$\begin{array}{lllll}\text { different } & \text { GJB1 } & \text { mutations } & \text { reported } & \text { so }\end{array}$ (http://hihg.med.miami.edu/code/http/cmt/public_html/index.html\#/; date last accessed December 2018) affecting all domains of $\mathrm{Cx} 32$, as well as the non-coding gene regions [14, 15]. Clinical series of CMT1X families with different mutations including complete deletion of the GJBI open reading frame show that patients have a similar phenotype and clinical course, indicating a loss of function mechanism underlying the disease $[1,16]$. Therefore, gene replacement therapy may potentially treat the disease. 
We have previously developed a gene delivery method by a single lumbar intrathecal injection for targeted expression in Schwann cells. Using a lentiviral vector carrying the GJB1 gene driven by the myelin protein zero $(\mathrm{Mpz})$ promoter we have shown that gene addition at the age of 2 months can rescue the demyelinating neuropathy in the Cx32 knockout (KO) model of CMT1X, either by direct intraneural [17] or by intrathecal injection [18]. These results have been replicated in 2month old $\mathrm{Cx} 32 \mathrm{KO}$ mice expressing the endoplasmic reticulum (ER)-retained T55I mutant associated with CMT1X [19]. However, in these studies we treated Cx32 KO mice before the onset of disease which occurs after 3 months of age [20-22]. Given the clinical course of the disease, therapeutic benefit from gene therapy needs to be also demonstrated after the onset of the neuropathy, as this would be highly relevant for treating patients with CMT1X with long established peripheral neuropathy.

Thus, in order to provide a clinically relevant proof of concept for the effectiveness of gene therapy for CMT1X, even after the onset of the disease, we delivered intrathecally a lentiviral vector carrying the human GJB1 gene into 6 month old Cx32 KO mice. This late intervention resulted in a widespread expression of $\mathrm{Cx} 32$ in lumbar roots and sciatic nerves of older mice with correct localization at the paranodal areas. Treatment trial initiated at 6 months of age resulted in increased motor performance that was maintained up to 10 months of age along with electrophysiological and morphological improvement. Our results confirm the efficacy of gene addition to provide therapeutic benefit in the model of CMT1X even after the onset of the neuropathy, supporting further the potential for this approach to treat CMT1X patients. 


\section{Materials and Methods}

\section{Cloning and production of lentiviral vectors}

Cloning of the lentiviral vectors and vector production and titration methods have been described in our previous studies $[17,18]$. Briefly, for the generation of Schwann-cell targeted lentiviral vectors the expression cassette was cloned into the lentivirus transfer vector pCCLsin.PPT.hPGK.GFP.pre by replacing the $P G K$ promoter with the rat myelin protein zero $(\mathrm{Mpz})$ promoter, which drives strongly gene expression in Schwann cells [23], and by downstream insertion of the human GJB1 open reading frame (ORF), along with the IRES.EGFP. A mock vector was also generated by cloning the $M p z$-IRES.EGFP expression cassette without the GJB1 ORF into the same lentiviral vector. Correct assembly of the expression cassettes was confirmed by restriction digest mapping and by sequencing the ORFs.

Recombinant lentiviruses were produced by transient co-transfection of HEK 293T cells with the transfer vector and the helper plasmids (CMVAR8.74 and pMD2-VSVG for pseudotyping) using the calcium phosphate co-precipitation method. A total of 5x106 293T cells were seeded in $10 \mathrm{~cm}$ plates $24 \mathrm{~h}$ prior to transfection in Iscove modified Dulbecco culture medium with $10 \%$ fetal bovine serum, penicillin $(100 \mathrm{IU} / \mathrm{ml})$, and streptomycin $(100 \mathrm{mg} / \mathrm{ml})$ in a $5 \% \mathrm{CO}_{2}$ incubator. One hour prior to transfection the culture medium was changed. A total of $64 \mu \mathrm{g}$ of plasmid DNA was used for the transfection per dish: $16 \mu \mathrm{g}$ of the envelope plasmid pMD2-VSVG, $16 \mu \mathrm{g}$ of the packaging plasmid CMV $\Delta \mathrm{R} 8.74$ and $32 \mu \mathrm{g}$ of the transfer vector plasmid. The precipitate was formed by adding the plasmids to a final volume of $540 \mu \mathrm{l}$ and $60 \mu \mathrm{l}$ of $2.5 \mathrm{M} \mathrm{CaCl}_{2}$ and then adding drop wise $600 \mathrm{ml}$ of 2x HEPES-buffered saline. The precipitate was added immediately to 
the cultures. The medium was then replaced after $6 \mathrm{~h}$ with fresh medium containing $1 \mathrm{mM} \mathrm{Na}$ butyrate. The conditioned medium was collected $60 \mathrm{~h}$ after transfection, cleared by low-speed centrifugation, and filtered through $0.22 \mu \mathrm{m}$-pore-size filters. The media was concentrated down from $6 \mathrm{ml}$ to $250 \mu \mathrm{l}$ volume by using the Lenti-X columns (CloneTech). The lentivirus titter was calculated using HIV-1 Gag p24 ELISA and one-step RT-qPCR for EGFP.

\section{Experimental Animals}

All intrathecal gene delivery experiments were conducted using 6-month-old mice Gjb1-null/Cx32 KO (C57BL/6_129) mice weighing 20-25 g, obtained from the European Mouse Mutant Archive, originally generated by Prof. Klaus Willecke (University of Bonn). In these mice, the neor gene was inserted in frame into the exon 2 of Gjbl gene which contains the ORF [24]. Both male and female mice were used in our experiments and showed no (sex-related) differences in their behavioral performance or nerve pathology. All experimental procedures in this study were conducted in accordance with animal care protocols approved by the Cyprus Government's Chief Veterinary Officer (project license CY/EXP/PR.L3/2017) according to national law, which is harmonized with EU guidelines (EC Directive 86/609/EEC).

\section{Intrathecal Vector Delivery}

We delivered the lentiviral vector as previously described $[18,25]$. Briefly, following a small skin incision along the lower lumbar spine level to visualize the spine, the lentiviral vector was delivered into the L5-L6 intervertebral space of anesthetized mice. A 50- $\mu$ L Hamilton syringe (Hamilton, Giarmata, Romania) connected to a 26-gauge needle was used to inject $30 \mu \mathrm{L}$ of lentiviral stock containing an estimated $4.0 \times 10^{11}-5.3 \times 10^{12}$ (full vector) and $7.5 \times 10^{11}-9.9 \times 10^{12}$ 
(mock vector) viral particles/mL. A flick of the tail was considered indicative of successful intrathecal administration.

\section{Immunofluorescence staining}

For immunostaining, mice were anesthetized with avertin according to institutionally approved protocols, and then transcardially perfused with normal saline followed by fresh $4 \%$ paraformaldehyde in $0.1 \mathrm{M}$ PB buffer. The lumbar-sacral spinal cords with spinal roots attached, as well as the bilateral sciatic and femoral motor nerves were dissected. All tissues were frozen for cryosections, while sciatic and femoral nerves were isolated and teased into fibers under a stereoscope. Teased fibers or sections were permeabilized in cold acetone and incubated at RT with a blocking solution of 5\% BSA (Sigma-Aldrich, Munich, Germany) containing 0.5\% Triton-

X (Sigma-Aldrich, Munich, Germany) for $1 \mathrm{~h}$. Primary antibodies used were: mouse monoclonal antibody against contactin-associated protein (Caspr, 1:50; gift of Dr Elior Peles, Weizmann Institute of Science), rabbit antisera against EGFP (1:1,000; Invitrogen, USA), Capr2 (1:200, Alomone Labs, Israel) and Cx32 (1:50; Sigma, Munich, Germany) all diluted in blocking solution and incubated overnight at $4{ }^{\circ} \mathrm{C}$. Slides were then washed in PBS and incubated with fluoresceinand rhodamine-conjugated mouse and rabbit cross-affinity purified secondary antibodies (1:500; Jackson ImmunoResearch,USA) for $1 \mathrm{~h}$ at RT. Cell nuclei were visualized with DAPI ( $1 \mu \mathrm{g} / \mathrm{ml}$; Sigma, Munich, Germany). Slides were mounted with fluorescent mounting medium and images photographed under a fluorescence microscope with a digital camera using Axiovision software (Carl Zeiss MicroImaging; Oberkochen, Germany). 


\section{Gene therapy study design}

The aim of this study was to examine whether a gene addition method can prevent the development of peripheral neuropathy in the CMT1X mouse model at a later stage after the onset of the neuropathy. The gene therapy trial was conducted using two groups of $\mathrm{Cx} 32 \mathrm{KO}$ mice. A minimum of 8-12 mice per treatment group for each outcome measure was considered adequate for assessing statistically significant differences based on our previous studies using similar models $[17,18]$. Animals were treated at the age of 6 months, after the onset of the pathology. Littermate mice were randomized to receive either LV-Mpz.GJBI (full) treatment or LV-Mpz.Egfp (mock treatment, as a control group) and were assigned a coding number for further identification. Mice were evaluated by behavioral testing before treatment, and again at the ages of 8 and 10 months, by an examiner blinded to the treatment condition, and used at the age of 10 months for electrophysiology or for quantitative morphometric analysis of semithin sections. Analysis of physiological and morphological results was also performed blinded to the treatment condition. Wild type (WT) mice of the same age were also evaluated by nerve conduction velocities and neuromuscular junction (NMJ) morphological analysis in order to compared with therapeutic benefit in treatment groups.

\section{Behavioral analysis}

Rotarod testing: Motor balance and coordination was determined as described previously [26] using an accelerating rotarod apparatus (Ugo Basile, Varese, Italy). Training of animals consisted of three trials per day with 15 -min rest period between trials, for 3 consecutive days. The mice were placed on the rod and the speed was gradually increased from 4 to 40 rotations per minute (rpm). Testing was performed on the fourth day using two different speeds, 20 and $32 \mathrm{rpm}$. Latency to fall was calculated for each speed. The test lasted until the mouse fell from the rod or after the 
mouse remained on the rod for $600 \mathrm{~s}$ and was then removed. Each mouse was placed on the rotarod three times at each speed used and three different values were obtained for each speed. Mean values were used for each mouse at the two different speeds.

Grip strength testing: To measure grip strength, mice were held by the tail and lowered towards the apparatus (Ugo Basile, Varese, Italy) until they grabbed the grid with the hind paws. Mice were gently pulled back until they released the grid. Measurements of the force in $\mathrm{g}$ were indicated on the equipment. Each session consisted of three consecutive trials and measurements were averaged. Hind limb force was compared between LV.Mpz-GJB1 and LV.Mpz-Egfp treated mice.

\section{Electrophysiological analysis}

Motor nerve conduction velocity (MNCV): $\mathrm{MNCV}$ was measured in vivo using published methods [27] from bilateral sciatic nerves following stimulation in anesthetized animals at the sciatic notch and distally at the ankle via bipolar electrodes with supramaximal square-wave pulses $(5 \mathrm{~V})$ of $0.05 \mathrm{~ms}$. The latencies of the compound muscle action potentials (CMAP) were recorded by a bipolar electrode inserted between digits 2 and 3 of the hind paw and measured from the stimulus artifact to the onset of the negative M-wave deflection. MNCV was calculated by dividing the distance between the stimulating and recording electrodes by the result of subtracting distal from proximal latency.

Quadriceps muscle contractility study: In order to assess in situ the function of the lumbar root and femoral motor axons we measured the contraction properties of the quadriceps muscle innervated by the femoral nerve in an anesthetized mouse as recently described [18]. After exposure of the motor part of the femoral nerve a stimulating hook electrode was used to stimulate the motor branch of the femoral nerve at $1 \mathrm{~Hz}$ using a constant current stimulator (DS3; Digitimer) 
with 5-6 mA and $200 \mu$ s duration pulse. The muscle contraction of the partially exposed quadriceps muscle was recorded with a force displacement transducer (FT03; Grass Technologies), which was attached to the muscle with a silk suture. The transducer was connected to a micromanipulator and for the experiment the muscle was extended $1 \mathrm{~mm}$ each time until the muscle contraction reached the maximum value. The average amplitude and duration of the force generated by the quadriceps muscle contraction was compared between treatment groups.

\section{Determination of the Neurofilament-light (NfL) levels}

Blood samples were obtained from 10-month old untreated Cx32KO, WT, fully treated and mock treated mice prior to sacrificing using standard methods [28]. Blood samples were taken and processed within one hour. Blood was collected into EDTA-containing tubes and centrifuged at $20^{\circ} \mathrm{C}$ at $3500 \mathrm{rpm}$ for 10 minutes. Plasma was aliquoted and stored at $-80^{\circ} \mathrm{C}$ until testing. Plasma sample NFL concentration was determined at UCL using a commercially available NF-Light kit on a Single molecule array (Simoa) HD-1 instrument (Quanterix, Lexington, MA) [29, 30].

\section{Assessment of neuromuscular junction denervation}

To further examine the results of gene therapy on distal nerve endings and muscle innervation, we evaluated neuromuscular junctions (NMJs) of quadriceps muscles of WT, mock and fully treated mice. Muscles were isolated from mice that were subjected to electrophysiological analysis and maintained in $4 \%$ PFA overnight. PFA was removed and muscles were washed 3 times with PBS and then maintained in $30 \%$ sucrose overnight. Muscles were frozen and cut into $25 \mu \mathrm{m}$ sections. Sections were permeabilized in cold acetone and incubated at RT with a blocking solution of 5\% 
BSA (Sigma-Aldrich, Munich, Germany) containing 0.5\% Triton-X (Sigma-Aldrich, Munich, Germany) for $1 \mathrm{~h}$ and incubated overnight at $4{ }^{\circ} \mathrm{C}$ with a mouse monoclonal antibody against neurofilament (SMI31; 1:1000; Abcam, Cambridge, MA) diluted in blocking solution. Slides were then washed in PBS and incubated with fluorescein-conjugated mouse cross-affinity purified secondary antibodies (1:500; Jackson ImmunoResearch, USA) along with bungarotoxin that binds to acetylcholine receptors on the postsynaptic membrane (1:100, Molecular Probes, USA) for $1 \mathrm{~h}$ at RT. Cell nuclei were visualized with DAPI ( $1 \mu \mathrm{g} / \mathrm{ml}$; Sigma, Munich, Germany) and slides were mounted with fluorescent mounting medium. Z-stack images were obtained using a fluorescence microscope (Nikon Eclipse Ni; Tokyo, Japan) with digital camera (DS-Qi2) using NIS-Elements software. Area, diameter and perimeter of the NMJs were measured using the Image Pro Plus software (version 6.0; Media Cybernetics) using a custom made macro. In addition, the ratios of NMJs that were devoid of innervation or partly innervated (missing completely or partly SMI31immunoreactive axons entering the NMJ area) were measured in at least 50 NMJs per individual mouse.

\section{Morphometric Analysis of myelination in Lumbar Roots and Peripheral Nerves}

Mice were transcardially perfused with $2.5 \%$ glutaraldehyde in $0.1 \mathrm{M} \mathrm{PB}$ buffer. The lumbar spinal cord with multiple spinal roots attached, as well as the femoral and sciatic nerves, were dissected and fixed overnight at $4{ }^{\circ} \mathrm{C}$, then osmicated, dehydrated, and embedded in araldite resin (all purchased from Agar Scientific, Essex, UK). Transverse semithin sections (1 $\mu \mathrm{m})$ of the lumbar spinal cord with roots and the middle portion of the femoral motor and sciatic nerves were obtained and stained with alkaline toluidine blue (Sigma-Aldrich, Munich, Germany). Sections were 
visualized with $10 \times, 20 \times$, and $40 \times$ objective lenses and captured with a Nikon DS-L3 camera (Nikon Eclipse-Ni; Tokyo, Japan). Images of whole root or transverse nerve sections were obtained at 100-200× final magnification, and a series of partially overlapping fields covering the cross-sectional area of the roots or the nerves were captured at $400 \times$ final magnification. These images were used to examine the degree of abnormal myelination in both groups as described

previously [17, 21, 31]. In brief, all demyelinated, remyelinated, and normally myelinated axons were counted using the following criteria: axons larger than $1 \mu \mathrm{m}$ without a myelin sheath were considered demyelinated, axons with myelin sheaths $<10 \%$ of the axonal diameter and/or axons surrounded by "onion bulbs" (i.e., circumferentially arranged Schwann cell processes and extracellular matrix) were considered remyelinated, and other myelinated axons were considered normally myelinated.

In addition, we counted the number of foamy macrophages present within the entire cross section of each root or nerve, as an indication of inflammation. Macrophages were identified in semithin sections at 400× magnification as cells laden with myelin debris, devoid of a basement membrane, and extending small, microvilli-like processes, as described previously [32, 33]. The macrophage count was calculated as the ratio per 1,000 myelinated fibers, to account for size differences between different spinal roots and nerves. All pathological analyses were performed blinded to the treatment condition of each mouse.

\section{Statistical analysis}

The percentages of Cx32-expressing paranodal myelin areas in immunostained spinal roots and sciatic nerves of different mutant lines injected with the full vector were compared with Student's 
t-test. Behavioral testing results as well as morphological analysis data including the proportion of abnormally myelinated fibers and the number of macrophages in lumbar spinal roots and peripheral nerves obtained from mock-treated and fully treated mice during the treatment trial were compared using the unpaired two-sided Student's t-test as well as the Mann-Whitney U test (significance level for all comparisons, $\mathrm{P}<0.05$ ) using Graphad Instat3 software (GraphPad, USA).

\section{Results}

\section{Virally induced expression of $G J B 1$ gene in 6 month-old $G j b 1$-null mice}

We have previously shown that lentiviral vector delivery of the human GJB1 gene in 2 month-old Cx32 KO mice, before the onset of peripheral neuropathy, results in stable expression of Cx32 [18]. In this study we examined the expression levels of the same vector following delivery to 6 month-old mice, after the onset of the disease. Expression levels of EGFP and Cx32 were determined 4 and 8 weeks post injection in lumbar roots and sciatic nerves. EGFP was detected in the perinuclear cytoplasm in a subset of Schwann cells in both the lumbar root sections (Fig. 1A-B) and in sciatic nerve teased fibers (Fig. 1C-D). Quantification of EGFP expression (Fig. 1E) 4 weeks post injection (at 7 months of age, in $n=4$ mice examined) showed that EGFP expression rates (\% of EGFP positive Schwann cells) reached $29.6 \pm 5.81 \%$ in lumbar roots and $42.7 \pm 2.61 \%$ in sciatic nerves, while at 8 weeks ( 8 months of age, $n=3$ mice examined) expression rates reached $21.0 \pm 2.02 \%$ in lumbar roots and $33.3 \pm 3.93 \%$ in sciatic nerves (data not shown). Differences between the two time points were not significant $(\mathrm{p}>0.05)$. 
Expression of Cx32 was also detected both at 4 and at 8 weeks after vector delivery in 6-mo old Cx32 KO PNS tissues, correctly localized to the paranodal areas of lumbar roots (Fig. 1G) and sciatic nerves (Fig. 1I) of mice injected with the full vector both at 4 and at 8 weeks post-injection, but was absent in non-injected littermates (Fig. 1F, H). Quantification of Cx32 expression rates 4 weeks post-injection ( $n=4$ mice examined) showed that $37.2 \pm 3.4 \%$ of paranodal areas identified by labeling with paranodal marker Caspr were Cx32-positive in lumbar roots and $43.5 \pm 6.18 \%$ in sciatic nerves (Fig. 1J). Cx32 expression rates remained stable at 8 weeks post-injection ( $\mathrm{n}=3$ mice examined) reaching $40.1 \pm 7.40 \%$ in lumbar roots and $42.8 \pm 0.62 \%$ in sciatic nerves (data no shown).

\section{Improvement of motor performance in treated 6 month-old $\mathrm{Cx32} \mathrm{KO}$ mice}

After confirming adequate vector expression in 6 month-old $\mathrm{Cx} 32 \mathrm{KO}$ mice with already advanced peripheral nerve pathology, we proceeded to the treatment trial. For this purpose mice were randomized into two groups, one receiving the mock and the other the full vector. Motor performance was assessed before the injection at the age of 6 months and again at the age of 8 ( 2 months post-injection) and 10 months (4 months post-injection) by rotarod (at 20 and 32 RPM) and foot grip tests. Rotarod analysis at the age of 6 months, at baseline before initiating the treatment with the lentiviral vector, showed as expected no differences between the two groups at either 20 or at 32 RPM (Suppl. Table 1 and Fig. 2A-B). Likewise, foot grip test showed no significant difference between the two groups (Fig. 2C).

At 2 months post injection, at the age of 8 months, there was a significant improvement in the fully treated group $(n=35)$ compared to the mock vector treated mice $(n=32)$ as indicated by the results 
of both rotarod and foot grip analysis. Fully treated mice remained on the rotarod significantly longer both at 20 and at 32 RPM (Suppl. Table 1 and Fig. 2D-E). Fully treated mice also generated a higher force in the foot grip test compared to mock treated mice (Suppl. Table 1 and Fig. 2F). The significant improvement of motor performance in the treatment group was maintained up to 4 months after treatment, at the age of 10 months. Although at the speed of 20 RPM the improvement in the full treatment group did not reach statistical significance, at 32 RPM they performed better than mock-treated littermates (Suppl. Table 1 and Fig. 2G-H). Moreover, 10month old fully treated $\mathrm{Cx} 32 \mathrm{KO}$ mice showed significantly higher force generation in the foot grip test compared to mock treated littermates (Table 1 and Fig. 2I).

Longitudinal comparison of the motor performance at different time points within each treatment group showed that fully treated mice improved significantly 2 months post-injection, from 6 to 8 months of age, and this improvement was maintained until 10 months of age (Fig. 2J-L and Suppl. Table 1). Although comparison between the baseline (6 month of age) and either 8 or 10 months of age showed improvement in all behavioral tests in treated mice, there was no significant change from 8 to 10 months of age, indicating that improvement occurred during the first months after treatment while motor performance remained stable thereafter without further improvement. In contrast, in the mock vector treated group there was no significant change in any of the behavioral tests between the time points examined (Fig. 2J-L and Suppl. Table 1).

\section{Improvement of sciatic nerve motor conduction velocity and quadriceps muscle contractility in late treated $\mathrm{Cx32} \mathrm{KO}$ mice}


Motor nerve conduction velocity (MNCV) of sciatic nerve was measured 4 months after treatment in order to assess functional properties in both groups. MNCV values were improved in the fully treated group reaching $37.0 \pm 1.29 \mathrm{~m} / \mathrm{s}(\mathrm{n}=18)$ while velocities in the mock group were $25.5 \pm 1.16$ $\mathrm{m} / \mathrm{s}(\mathrm{n}=12)($ Fig. 3A; $\mathrm{p}<0.0001)$. MNCV values of the fully treated group were close to those of the WT mice which reached 41.7 $\pm 1.62(n=8 ; p>0.05)$. Further to the MNCV, we also measured the compound muscle action potential (CMAP) amplitude in both groups and found a trend for higher amplitudes in treated mice which reached $2.4 \pm 0.22 \mathrm{mV}(\mathrm{n}=18)$ compared to the mock group that reached $2.0 \pm 0.22 \mathrm{mV}(\mathrm{n}=12)$, although not statistically significant (Fig. 3B; $\mathrm{p}>0.05)$. Sciatic CMAP amplitudes of treated mice remained lower than those of WT mice which reached $3.3 \pm 0.29$ $\mathrm{mV}(\mathrm{n}=8 ; \mathrm{p}=0.0259)$.

Furthermore, we analyzed the quadriceps muscle contractility after stimulation of the femoral motor nerve in situ as previously described [18]. Both force and duration of contraction were used to assess functional improvement. This analysis showed that force and duration were improved in the fully treated mice compared to the mock group $(\mathrm{p}<0.05)$. The highest mean values were observed at a $6 \mathrm{~mm}$ extension in all cases with the highest value for mock treated mice at $0.27 \pm 0.02$ $\mathrm{N}(\mathrm{n}=9$ mice) and for fully treated at $0.34 \pm 0.02 \mathrm{~N}(\mathrm{n}=10)$ (Fig. 3C). The mean duration of quadriceps contraction (Fig. 3D) reached $73.3 \pm 3.07 \mathrm{~ms}$ in mock treated mice $(\mathrm{n}=9)$ while the duration of the fully treated mice reached $85.3 \pm 4.61 \mathrm{~ms}(\mathrm{n}=10 ; \mathrm{p}<0.05)$. Thus, electrophysiological studies with emphasis on motor fibers that are predominantly affected in this CMT1X model showed significant improvement following post-onset gene therapy.

\section{Improved neuromuscular junction (NMJ) innervation in treated mice}


NMJ innervation was measured in sections of 10-month old WT (Fig. 4A) as well as of mock (Fig. 4B) compared to full vector treated (Fig. 4C) Cx32 KO mice. Partial or completely lost innervation was frequently observed in $\mathrm{Cx} 32 \mathrm{KO}$ quadriceps muscle with bungarotoxin-labelled endplates partially or completely devoid of axon terminals, but these abnormalities were improved in treated mice. Quantification confirmed improved NMJ innervation in fully treated compared to mock treated mice although the values of fully treated mice did not reach the WT levels. The percentage of fully innervated NMJs showed a trend of increase in the fully treated mice $(34.9 \pm 6.73 \%$; $=471$ NMJs from 6 mice) compared to the mock group (19.4 $55.54 \%$; $=320$ NMJs from 5 mice) but this improvement was not statistically significant $(\mathrm{p}>0.05)$ and did not reach the NMJ innervation rates of WT mice $(97.3 \pm 1.10 \%, \mathrm{p}<0.0001 ; \mathrm{n}=206 \mathrm{NMJ}$ from 4 mice; Fig. 4D). However, the rate of the partially innervated NMJs was increased in fully treated mice $(37.3 \pm 1.21 \%)$ compared to the mock group $(24.9 \pm 2.62 \%$; $=0.0014$; Fig. 4E) while completely denervated NMJs were more frequent in the mock group $(53.2 \pm 7.67 \%)$ compared to fully treated mice $(28 \pm 6.23 \% ; \mathrm{p}=0.0299$; Fig. 4F).

Furthermore, measurement of area, diameter and perimeter of the NMJs in all groups showed that both area and diameter were increased in the fully treated compared to the mock group. Average NMJ area in the mock group was $385.6 \pm 12.11 \mu \mathrm{m}^{2}$ compared $416 \pm 10.00 \mu \mathrm{m}^{2}$ in the treated group (p=0.0239; Fig. 4G). Likewise, we observed a small increase in the diameter of the NMJs (mock group: $20.3 \pm 0.29 \mu \mathrm{m}$; treated group: $21.3 \pm 0.22 \mu \mathrm{m}$; p=0.0036; Fig. 4H). In contrast, the NMJ perimeter did not significantly differ between the two groups $(119.7 \pm 3.34 \mu \mathrm{m}$ in mock and 110.6 $\pm 2.11 \mu \mathrm{m}$ in treated group, $\mathrm{p}>0.05$; Fig. 4I). Overall, these findings indicate improved 
although not completely restored muscle innervation at 10 months of age following gene therapy after onset in $\mathrm{Cx} 32 \mathrm{KO}$ mice.

\section{Determination of Neurofilament-light (NfL) levels}

Recent studies have shown an elevation of serum NfL levels in patients with different CMT types including CMT1X compared to controls, as well as a correlation between elevated concentrations and disease severity, suggesting that this may be a useful biomarker for assessing disease progression and potential response to treatment in future clinical trials [30]. In order to clarify whether the Cx32 KO model of CMT1X shows an elevation of NfL levels, we first examined blood samples obtained from 10-month-old untreated $\mathrm{Cx} 32 \mathrm{KO}$ and WT littermates. This comparison confirmed a significant elevation of NfL levels in $\mathrm{Cx} 32 \mathrm{KO}$ mice $(\mathrm{n}=10)$ reaching $328.0 \pm 24.94 \mathrm{pg} / \mathrm{ml}$ (range: 222 to 481.2 ) compared to the levels of WT mice ( $\mathrm{n}=8)$ reaching 111.5 $\pm 26.77 \mathrm{pg} / \mathrm{ml}$ (range: 34.8 to 266.4), a 2.9-fold elevation (Fig. 5A; $<<0.00001$ ). Having confirmed that serum NFL is a biomarker in this neuropathy model, we then compared NfL levels in 10-month old Cx32 KO mice that were treated at the age of 6 months, compared to mock vector treated littermates. This analysis showed a significant reduction of NfL levels in the full treatment group ( $\mathrm{n}=5)$ by 1.5 fold ( $268.9 \pm 6.47 \mathrm{pg} / \mathrm{ml}$; range: 251.5 to 281.6$)$ compared to the mock treatment group (407.2 $\pm 21.47 \mathrm{pg} / \mathrm{ml}$; $\mathrm{n}=8$; range: 334.7 to 536.4 ) (Fig. $\mathbf{5 B}$; $\mathrm{p}=0.0016$ ). Thus, serum NfL levels appear to be also a treatment responsive biomarker for CMT1X and supports the findings of improved motor function and muscle innervation following gene therapy.

Improved pathology in lumbar roots and peripheral nerves after delayed treatment in $\mathrm{Cx} 32$ KO mice 
Morphological analysis was performed in transverse sections of anterior lumbar roots, mid-sciatic and femoral motor nerves of 10-month-old Cx32 KO mice injected either with the LV-Mpz-GJB1 or the mock vector. Multiple roots, as well as bilateral nerves when available, were examined, and results were averaged per mouse. The number of abnormally myelinated fibers, including demyelinated and remyelinated fibers, was counted and their proportion to the total number of fibers was calculated [17-19]. Foamy macrophages were also counted [18], and their numbers per 1,000 myelinated fibers were compared (to account for variations in root and nerve size).

In the anterior lumbar roots of the fully treated mice ratio of abnormally myelinated fibers was reduced compared to the mock group (Fig. 6; Suppl. Table 2). The ratio of abnormal fibers was $0.156 \pm 0.01$ in the fully treated mice $(n=10)$, compared with $0.259 \pm 0.03$ in the mock-treated mice ( $\mathrm{n}=10 ; \mathrm{p}=0.0011$, Mann-Whitney $U$ test). Likewise, macrophage numbers were lower in the anterior roots of fully treated $(6.42 \pm 0.85 / 1,000$ fibers) compared to mock-treated mice $(9.75 \pm$ $0.64 / 1,000$ fibers; $\mathrm{p}=0.0052$ ).

Likewise, in mid-sciatic nerves, the ratios of abnormally myelinated fibers were reduced in fully treated mice compared to the mock group (Fig. 7; Suppl. Table 3). The ratio of abnormal sciatic nerve fibers was $0.064 \pm 0.003$ in fully treated $(n=10)$ compared to $0.086 \pm 0.006$ in mock-treated mice ( $\mathrm{n}=10 ; \mathrm{p}=0.0089$, Mann-Whitney $U$ test). Improvement was also evident in the reduction of foamy macrophages, the numbers of which reached 3.0 $\pm 0.25 / 1,000$ fibers in the fully treated mice compared to $4.5 \pm 0.46 / 1,000$ fibers in mock group ( $\mathrm{p}=0.0232)$. 
Finally, improvement of myelination was observed also in femoral motor nerves (Fig. 8; Suppl.

Table 4). The ratio of abnormally myelinated fibers was $0.239 \pm 0.02$ in the fully treated group compared to $0.332 \pm 0.02$ in the mock group $(\mathrm{p}=0.0052)$. However, the numbers of macrophages were not significantly reduced in the fully treated mice $(4.3 \pm 0.88 / 1,000$ fibers $)$ compared to the mock group $(6.1 \pm 0.88 / 1,000$ fibers; $p>0.05)$.

\section{Discussion}

In the current study we used a lentiviral vector-mediated gene replacement therapy targeting Schwann cells to treat for the first time post-onset Cx32 KO mice, the mouse model for CMT1X. Intrathecal delivery of the viral vector carrying the GJB1 gene in 6-month old $\mathrm{Cx} 32$ deficient mice resulted in widespread expression of the virally delivered $\mathrm{Cx} 32$ in peripheral nervous system tissues, and provided a significant therapeutic benefit in this model of demyelinating neuropathy.

In our previous studies using the $\mathrm{Cx} 32 \mathrm{KO}$ model of CMT1X we have treated animals before the onset of the demyelinating neuropathy which occurs around 3 months of age [20-22] and may be preceded by axonal dysregulation as early as at 2 months of age [31]. This pre-onset treatment resulted in widespread vector distribution in the PNS and GJB1 gene expression, with rescue of the demyelinating neuropathy $[18,19]$. In this study, although we treated 6-month-old mice, well after the onset of peripheral neuropathy, we found a similar widespread vector distribution and Cx32 expression rates as that observed in 2 month old mice [18]. This is an indication that gene replacement targeting myelinating Schwann cells can be efficient even after the onset of the neuropathy regardless of age, at least in the mouse model of CMT1X. Gene expression was also restricted to myelinating Schwann cells as in the 2-month-old animals, which is an important 
aspect of CMT1X gene therapy strategy since in the PNS Cx32 is specifically expressed in myelinating Schwann cells where it forms gap junction channels through the non-compact myelin layers [34].

This late treatment study in the CMT1X model provided also further insights into the course of the disease. Behavioral analysis showed that mock-treated $\mathrm{Cx} 32 \mathrm{KO}$ mice remained rather stable over time after 6 months of age with no further progression, while fully treated mice showed significant improvement initially, that was maintained until the end of the study period. This indicates that in Cx32 KO mice functional deficits occur before 6 months of age and remain stable over time, perhaps a result of an equilibrium between de- and re-myelination and associated axonal degeneration. This may be a limitation of this neuropathy model as it does not fully reproduce the steady progression of the disease in patients with CMT1X, characterized by progressive neuropathy that worsens over time $[1,3,35]$ mainly due to accumulating axonal loss [36, 37]. Nevertheless, post-onset response in the CMT1X mouse model with functional improvement in treated mice indicates that expression of $\mathrm{Cx} 32$ can reverse some of the pathological changes already present, leading to an improvement of motor performance within the first two months after gene delivery that is maintained up to 10 months of age.

Improved motor performance in late treated $\mathrm{Cx} 32 \mathrm{KO}$ mice correlated with improvement in electrophysiological properties. MNCVs were increased in LV-Mpz.GJB1 compared to LV$M p z . E g f p$ (mock)-treated mice at 10 months of age, similar to our observations in pre-onset treated mice [18] . These results are also in accordance with the findings of Duque et al. (2016) [38], showing similar improvement of the CMAP amplitude in pre- and post-symptomatic animals of 
spinal muscular atrophy (SMA) treated with the AAV9 viral vector. We found that mock-treated 10-month old Cx32 deficient mice in our study had lower MNCVs than previously studied untreated Cx32 KO mice at about the same age [20, 24, 32]. This may be a side effect of the injection itself. LV.Mpz.GJB1 late treated Cx32 KO mice showed significantly higher MNCV and improved quadriceps muscle contractility at 10 months of age, similar to the improvements observed in treated Cx32 KO mice prior to the onset of the neuropathy [18]. Although we found a trend towards improvement of the CMAP amplitude in treated mice as well, this did not reach statistical significance. This may be related to the fact that decreased CMAP amplitudes in initially characterized $\mathrm{Cx} 32 \mathrm{KO}$ mice were only detectable at the age of 6 month and actually reached almost WT levels by 1 year of age [20], perhaps secondary to regenerating units during the course of the disease. Finally, quadriceps muscle contractility was significantly improved in this model with predominantly motor neuropathy [20, 22].

NMJ evaluation was in accordance with the results of electrophysiological analysis, since morphological analysis of NMJ innervation in the quadriceps muscle, which was also used in electrophysiological analysis, showed that fully treated mice presented higher rates of NMJ innervation although the innervation was mainly partial in contrast to the mock group where most of the NMJs were denervated. Our results of NMJ analysis at the age of 10 months are similar to Groh et al. (2010) [32] showing low percentages of denervation in the Cx32 KO mouse and almost no denervation in the WT mice. A more detailed analysis performed in our study demonstrated also the percentage of fully and partially innervated NMJs in this model. Comparison of the Cx32 KO mouse NMJ results and a recently published analysis of NMJ pathology in the CMT4C mouse model, shows similarities in NMJ area, diameter and perimeter but the CMT4C model shows an 
increase in post-synaptic fragmentation [39], that was not evaluated in our study, but may be indirectly reflected in the tendency for larger NMJ diameter in the mock compared to the fully treated $\mathrm{Cx} 32 \mathrm{KO}$ or the WT group.

Our morphological analysis showed reduction in the abnormally myelinated fibers in all PNS tissues examined, as well as in the numbers of foamy macrophages although the latter change did not reach statistical significance in femoral motor nerves. Although our results indicate morphological improvement and are similar to the animals treated before the onset of the neuropathy [18], the mean values of both abnormally myelinated fibers and foamy macrophages were increased by about $60 \%$ in both late-treated groups compared to early-treated groups in most PNS tissues examined, including the anterior lumbar roots and femoral motor nerves. Only in sciatic nerves the ratios of abnormal fibers were similar in both late and early-treated groups, likely reflecting the fact that neuropathy in this model affects predominantly the motor fibers $[20,40]$ causing progressive dysfunction, while the sciatic nerve is mixed including both motor and sensory fibers and shows minimal progression, making it less indicative of a therapeutic response. Thus, overall there was an improvement of pathology in peripheral nerves and lumbar roots of late treated Cx32 KO mice, although not to the degree we observed with pre-onset treatment and morphometric analysis at 8 months of age $[18,19]$. Similar observations have been reported in other disease models including the SMA gene therapy study in which both pre-symptomatic and post-symptomatic groups showed an improvement in the morphological properties, but postsymptomatic treatment was less effective compared to pre-symptomatic treatment [38]. Early intervention was also more effective in diabetic neuropathy models compared to late intervention [41]. 
Further to the demonstration of functional and morphological improvements following post-onset treatment, we also studied blood Nfl concentration in our treatment groups as a clinically relevant biomarker that correlates with peripheral nerve degeneration and was found to be increased in patients with CMT1X and other CMT types [30]. We demonstrate for the first time that blood Nfl levels are elevated in 10-month old $\mathrm{Cx} 32 \mathrm{KO}$ mice compared to WT controls, confirming that this is a useful biomarker in this model of CMT1X. Furthermore, we found a significant therapeutic response with decreased NfL concentrations in treated compared to mock treated mice, indicating that gene replacement therapy can ameliorate pathological changes affecting the axon itself reducing axonal degeneration, even with post-onset treatment. This biomarker becomes even more relevant for CMT1X because studies both in patient biopsies [36], as well as in the Cx32 KO model $[31,42]$ indicate a direct negative effect of $\mathrm{Cx} 32$ loss on axonal cytoskeleton and neurofilament phosphorylation and function, that likely drives axonal degeneration along with demyelination. Thus, blood Nfl levels can serve both as a diagnostic as well as treatment-response biomarker in pre-clinical and clinical studies focusing on CMT1X therapies.

It is difficult to correlate precisely the timing of our intervention in the $\mathrm{Cx} 32 \mathrm{KO}$ mouse model with the stages and clinical course of the neuropathy in CMT1X patients. There is a well-described age-depended loss of myelinated fibers in biopsied nerves of CMT1X patients [35, 43-45] leading to a progressive loss of motor units that correlates with clinical disability [37], while slowing of NCV may be present already in pre-symptomatic children and is less indicative of neurological deficits $[46,47]$. Thus, post-onset response in the mouse may not be entirely predictive of a late treatment response in patients, for example after childhood and adolescence, when axonal loss 
progresses. On the other hand, the ongoing formation of axonal sprouts along with remyelination as a mechanism of regeneration have been recognized as major features both in CMT1X patient biopsies as well as in the mouse model, indicating that by resorting Cx32 expression in viable Schwann cells one would expect a therapeutic benefit in patients even in adulthood.

Another limitation of our study is that biodistribution and expression rates of the viral vectors obtained in the mouse model may not be fully reproduced in larger animals or in humans, and this remains to be shown. Although lentiviral vectors have a well demonstrated tropism for myelinating Schwann cells $[17,18]$, including human [48], their direct in vivo application may be limited by their ability to integrate into the host genome, creating possible insertional mutagenesis risks. Alternative vectors such as adeno-associated vectors that remain episomal may offer an advantage, if serotypes with low immunogenicity and adequate tropism for human myelinating Schwann cells are identified.

In conclusion, this study provides evidence that late, post-onset gene replacement therapy targeting myelinating Schwann cells can partly reverse the combination of demyelinating neuropathy and axonal degeneration in the CMT1X mouse model resulting in significant functional and morphological improvements. Although late treatment provides a clear therapeutic benefit, our results indicate that treatment should be pursuit as early in the disease course as possible, in order to achieve the highest possible therapeutic response.

\section{Funding:}

This study was co-funded by the Muscular Dystrophy Association and Charcot-Marie-Tooth Association (Grants 480030 and 603003 to KAK). AK is a recipient of a Young Researcher Award 
CULTURE/BR-NE/0416/07 financed by the Republic of Cyprus through the Research Promotion Foundation.

Acknowledgements: We thank Dr Elior Peles (Weizmann Institute of Science) for the Caspr antibody and Dr Kyriacos Kyriacou for advice on processing semithin sections. We also thank Martha Foiani for help with measuring NfL samples.

\section{References}

1. Shy, M.E., et al., CMTIX phenotypes represent loss of GJB1 gene function. Neurology, 2007. 68(11): p. 849-55.

2. Abrams, C.K. and S.S. Scherer, Gap junctions in inherited human disorders of the central nervous system. Biochim Biophys Acta, 2012. 1818(8): p. 2030-47.

3. Kleopa, K.A. and S.S. Scherer, Molecular genetics of X-linked Charcot-Marie-Tooth disease. Neuromolecular Med, 2006. 8(1-2): p. 107-22.

4. $\quad$ Kleopa, K.A., et al., Phenotypic and cellular expression of two novel connexin32 mutations causing CMT1X. Neurology, 2006. 66(3): p. 396-402.

5. Paulson, H.L., et al., Transient central nervous system white matter abnormality in $X$ linked Charcot-Marie-Tooth disease. Ann Neurol, 2002. 52(4): p. 429-34. 
6. Taylor, R.A., et al., The CNS phenotype of X-linked Charcot-Marie-Tooth disease: more than a peripheral problem. Neurology, 2003. 61(11): p. 1475-8.

7. Lu, Y.Y., et al., Clinical and Genetic Features of Chinese X-linked Charcot-Marie-Tooth Type 1 Disease. Chin Med J (Engl), 2017. 130(9): p. 1049-1054.

8. Saporta, A.S., et al., Charcot-Marie-Tooth disease subtypes and genetic testing strategies. Ann Neurol, 2011. 69(1): p. 22-33.

9. Liang, C., et al., Axonal excitability in X-linked dominant Charcot Marie Tooth disease. Clin Neurophysiol, 2014. 125(6): p. 1261-9.

10. Martikainen, M.H. and K. Majamaa, Novel GJB1 mutation causing adult-onset CharcotMarie-Tooth disease in a female patient. Neuromuscul Disord, 2013. 23(11): p. 899-901.

11. Jerath, N.U., et al., Charcot-marie-tooth disease type $1 X$ in women: Electrodiagnostic findings. Muscle Nerve, 2016. 54(4): p. 728-32.

12. Bruzzone, R., T.W. White, and D.L. Paul, Connections with connexins: the molecular basis of direct intercellular signaling. Eur J Biochem, 1996. 238(1): p. 1-27.

13. Balice-Gordon, R.J., L.J. Bone, and S.S. Scherer, Functional gap junctions in the schwann cell myelin sheath. J Cell Biol, 1998. 142(4): p. 1095-104.

14. Murphy, S.M., et al., A novel mutation in the nerve-specific 5'UTR of the GJB1 gene causes X-linked Charcot-Marie-Tooth disease. J Peripher Nerv Syst, 2011. 16(1): p. 65-70.

15. Tomaselli, P.J., et al., Mutations in noncoding regions of GJB1 are a major cause of Xlinked CMT. Neurology, 2017. 88(15): p. 1445-1453.

16. Panosyan, F.B., et al., Cross-sectional analysis of a large cohort with X-linked CharcotMarie-Tooth disease (CMTX1). Neurology, 2017. 89(9): p. 927-935.

17. Sargiannidou, I., et al., Intraneural GJBI gene delivery improves nerve pathology in a model of X-linked Charcot-Marie-Tooth disease. Ann Neurol, 2015. 78(2): p. 303-16.

18. Kagiava, A., et al., Intrathecal gene therapy rescues a model of demyelinating peripheral neuropathy. Proc Natl Acad Sci U S A, 2016. 113(17): p. E2421-9.

19. Kagiava, A., et al., Intrathecal gene therapy in mouse models expressing CMT1X mutations. Hum Mol Genet, 2018. 27(8): p. 1460-1473.

20. Anzini, P., et al., Structural abnormalities and deficient maintenance of peripheral nerve myelin in mice lacking the gap junction protein connexin 32. J Neurosci, 1997. 17(12): p. 4545-51. 
21. Sargiannidou, I., et al., Connexin32 mutations cause loss of function in Schwann cells and oligodendrocytes leading to PNS and CNS myelination defects. J Neurosci, 2009. 29(15): p. 4736-49.

22. Scherer, S.S., et al., Connexin32-null mice develop demyelinating peripheral neuropathy. Glia, 1998. 24(1): p. 8-20.

23. Scherer, S.S., et al., Transgenic expression of human connexin32 in myelinating Schwann cells prevents demyelination in connexin32-null mice. J Neurosci, 2005. 25(6): p. 1550-9.

24. Nelles, E., et al., Defective propagation of signals generated by sympathetic nerve stimulation in the liver of connexin32-deficient mice. Proc Natl Acad Sci U S A, 1996. 93(18): p. 9565-70.

25. Kagiava, A. and K.A. Kleopa, Intrathecal Delivery of Viral Vectors for Gene Therapy. Methods Mol Biol, 2018. 1791: p. 277-285.

26. Savvaki, M., et al., The expression of TAG-1 in glial cells is sufficient for the formation of the juxtaparanodal complex and the phenotypic rescue of tag-1 homozygous mutants in the CNS. J Neurosci, 2010. 30(42): p. 13943-54.

27. Zielasek, J., R. Martini, and K.V. Toyka, Functional abnormalities in PO-deficient mice resemble human hereditary neuropathies linked to P0 gene mutations. Muscle Nerve, 1996. 19(8): p. 946-52.

28. Parasuraman, S., R. Raveendran, and R. Kesavan, Blood sample collection in small laboratory animals. J Pharmacol Pharmacother, 2010. 1(2): p. 87-93.

29. Rohrer, J.D., et al., Serum neurofilament light chain protein is a measure of disease intensity in frontotemporal dementia. Neurology, 2016. 87(13): p. 1329-36.

30. Sandelius, A., et al., Plasma neurofilament light chain concentration in the inherited peripheral neuropathies. Neurology, 2018. 90(6): p. e518-e524.

31. Vavlitou, N., et al., Axonal pathology precedes demyelination in a mouse model of X-linked demyelinating/type I Charcot-Marie Tooth neuropathy. J Neuropathol Exp Neurol, 2010. 69(9): p. 945-58.

32. Groh, J., et al., Attenuation of MCP-1/CCL2 expression ameliorates neuropathy in a mouse model for Charcot-Marie-Tooth 1X. Hum Mol Genet, 2010. 19(18): p. 3530-43. 
33. Kobsar, I., et al., Preserved myelin integrity and reduced axonopathy in connexin32deficient mice lacking the recombination activating gene-1. Brain, 2003. 126(Pt 4): p. 80413.

34. Scherer, S.S., et al., Connexin32 is a myelin-related protein in the PNS and CNS. J Neurosci, 1995. 15(12): p. 8281-94.

35. Hahn, A.F., et al., X-linked dominant hereditary motor and sensory neuropathy. Brain, 1990. 113 ( Pt 5): p. 1511-25.

36. Hahn, A.F., et al., Pathological findings in the $x$-linked form of Charcot-Marie-Tooth disease: a morphometric and ultrastructural analysis. Acta Neuropathol, 2001. 101(2): p. 129-39.

37. Krajewski, K.M., et al., Neurological dysfunction and axonal degeneration in CharcotMarie-Tooth disease type 1A. Brain, 2000. 123 ( Pt 7): p. 1516-27.

38. Duque, S.I., et al., A large animal model of spinal muscular atrophy and correction of phenotype. Ann Neurol, 2015. 77(3): p. 399-414.

39. Cipriani, S., et al., Neuromuscular Junction Changes in a Mouse Model of Charcot-MarieTooth Disease Type 4C. Int J Mol Sci, 2018. 19(12).

40. Scherer, S.S. and L. Wrabetz, Molecular mechanisms of inherited demyelinating neuropathies. Glia, 2008. 56(14): p. 1578-89.

41. Yorek, M.S., et al., Early vs. late intervention of high fat/low dose streptozotocin treated C57Bl/6J mice with enalapril, alpha-lipoic acid, menhaden oil or their combination: Effect on diabetic neuropathy related endpoints. Neuropharmacology, 2017. 116: p. 122-131.

42. Sahenk, Z. and L. Chen, Abnormalities in the axonal cytoskeleton induced by a connexin32 mutation in nerve xenografts. J Neurosci Res, 1998. 51(2): p. 174-84.

43. Birouk, N., et al., X-linked Charcot-Marie-Tooth disease with connexin 32 mutations: clinical and electrophysiologic study. Neurology, 1998. 50(4): p. 1074-82.

44. Nicholson, G. and J. Nash, Intermediate nerve conduction velocities define X-linked Charcot-Marie-Tooth neuropathy families. Neurology, 1993. 43(12): p. 2558-64.

45. Vital, A., et al., Histopathological features of X-linked Charcot-Marie-Tooth disease in 8 patients from 6 families with different connexin32 mutations. J Peripher Nerv Syst, 2001. 6(2): p. $79-84$. 
46. Kuntzer, T., et al., Phenotypic expression of a Pro 87 to Leu mutation in the connexin 32 gene in a large Swiss family with Charcot-Marie-Tooth neuropathy. J Neurol Sci, 2003. 207(1-2): p. 77-86.

47. Vondracek, P., et al., X-linked Charcot-Marie-Tooth disease: phenotypic expression of a novel mutation Ile127Ser in the GJB1 (connexin 32) gene. Muscle Nerve, 2005. 31(2): p. $252-5$.

48. Hoyng, S.A., et al., Gene delivery to rat and human Schwann cells and nerve segments: $a$ comparison of AAV 1-9 and lentiviral vectors. Gene Ther, 2015. 22(10): p. 767-80. 